\title{
An Examination of the factors which may affect the duration of admission to the hospital of panic diagnosed patients during and pre-COVID-19 pandemic
}

\author{
gizem ISSIN ${ }^{1}$, Mehmet Gamsizkan² ${ }^{2}$ Ersin Tural ${ }^{3}$, Diren Cagatay ${ }^{1}$, and Ferda Keskin \\ Çimen $^{1}$ \\ ${ }^{1}$ Erzincan Binali Yildirim University, Mengucek Gazi Training and Research Hospital \\ 2 Duzce University, School of Medicine, Düzce, Turkey \\ ${ }^{3}$ Sultan Abdülhamid Han Training and Research Hospital
}

April 3, 2021

\begin{abstract}
Background: The present study aimed to determine the duration of hospital admission of the panic diagnosed patients, examine the factors that may influence hospital admission time, and identify the impact of the COVID-19 pandemic on hospital admission time. Materials and methods: In this study, the panic diagnosed patients between January 2018 and January 2021 were determined. These patients' demographic, clinical, and panic diagnosis form data were documented. The duration of hospital admission of patients during and pre-COVID-19 pandemic period was determined. Results: There were 65 panic diagnosed cases, of which one One patient had leukocytoclastic vasculitis, 10 patients had uterine contents without villi or trophoblasts, and 54 patients had unexpected malignancy. The mean time of admission to the hospital of verbally informed and not verbally informed cases were five days and 156 days, respectively in the pre-COVID group. All cases in the COVID pandemic group were verbally informed about panic diagnosis by phone call. The mean time (day) of admission to the hospital was 18.3 days (1-40). Admission times were on mean about 13.3 days longer in verbally informed cases in the COVID pandemic group compared to verbally informed cases in the pre-COVID group. Conclusion: We determined a dramatic decrease in the number of panic diagnosed cases during the COVID pandemic and patients who are verbally informed admitted to the hospital in a shorter time. Integrations of hospital panic diagnosis notification systems to health application programs and primary responsible family physician's systems may be useful for preventing unwanted delays.
\end{abstract}

An Examination of the factors which may affect the duration of admission to the hospital of panic diagnosed patients during and pre-COVID-19 pandemic

Running title: hospital admission time of panic diagnosed patient

\section{Abstract}

Aim/Background: The present study aimed to determine the duration of hospital admission of the panic diagnosed patients, examine the factors that may influence hospital admission time, and identify the impact of the COVID-19 pandemic on hospital admission time.

Materials and methods: In this study, the panic diagnosed patients between January 2018 and January 2021 were determined. These patients' demographic, clinical, and panic diagnosis form data were documented. The duration of hospital admission of patients during and pre-COVID-19 pandemic period was determined. 
Results: There were 65 panic diagnosed cases, of which one patient had leukocytoclastic vasculitis, 10 patients had uterine contents without villi or trophoblasts, and 54 patients had unexpected malignancy. The mean time of admission to the hospital of verbally informed and not verbally informed cases were five days and 156 days, respectively in the pre-COVID group. All cases in the COVID pandemic group were verbally informed about panic diagnosis by phone call. The mean time (day) of admission to the hospital was 18.3 days (1-40). Admission times were on mean about 13.3 days longer in verbally informed cases in the COVID pandemic group compared to verbally informed cases in the pre-COVID group.

Conclusion: We determined a dramatic decrease in the number of panic diagnosed cases during the COVID pandemic and patients who are verbally informed admitted to the hospital in a shorter time. Integrations of hospital panic diagnosis notification systems to health application programs and primary responsible family physician's systems may be useful for preventing unwanted delays.

Keywords: Panic diagnosis, unexpected diagnosis, significant diagnosis, critical value

\section{What's already known about this topic?}

Same pathology reports contain critical information about life-threatening changes that need immediate treatment.

Failure to follow up on the results of these reports or lack of appropriate communication of these reports results may lead to a delay in diagnosis that may cause severe or irreparable harm and may affect the patient outcome

\section{What does this article add?}

We observed that even if communication between the clinician and the pathologist is established in a brief time, in some cases, reaching the patient may take longer.

Most of the patients did not follow their pathology reports that may lead to delayed treatment and worsening of prognosis.

Our results revealed that receiving verbal phone notification was significantly associated with patients' admission to the hospital time.

To improve patient safety; informing patients verbally should be included in panic diagnosis policies, patients should be informed about the follow-up of the pathology report and their contact information should be kept up to date.

\section{Introduction}

Pathology reports are a crucial medical document that contains critical information about diagnosis, prognosis, and treatment. Although all pathology reports contain important information, some of them contain critical information about life-threatening changes that need immediate treatment. ${ }^{1}$ These diagnoses are considered panic diagnoses in surgical pathology. ${ }^{2}$ Failure to follow up on the results of these reports or lack of appropriate communication of these reports results may lead to a delay in diagnosis that may cause severe or irreparable harm and may affect the patient outcome. ${ }^{3}$ To ensure patient safety and prevent this delay, national pathology societies recommend that each pathology department should identify potential panic (unexpected) diagnosis lists and draw up a communication policy. ${ }^{2}$

The College of American Pathologists (CAP) evaluates panic (unexpected) diagnoses under Urgent Diagnoses and Significant, Unexpected Diagnoses titles. CAP defines urgent diagnoses as an important or life-threatening medical condition that requires urgent intervention and recommends that direct verbal communication occurs on the day of diagnosis. They also define Significant, Unexpected Diagnoses as a clinically unusual or unpredictable medical condition that needs to be addressed at some point in the patient's course and recommends that communication occurs as soon as possible. ${ }^{2}$ The Federation of Turkish pathology society considers Urgent, Significant, and unexpected diagnoses under a single title as a panic diagnosis. 
Several studies indicated that immediately effective verbal communication had a beneficial impact on patient's outcome and treatment management. ${ }^{4,5}$ Although communication between the clinician and the pathologist is established in a brief time, in some cases, reaching the patient may take longer. In the case of patients with an unexpected malignancy, prolonged hospital admission time may result in delayed treatment and worsening of prognosis.

The severe acute respiratory syndrome coronavirus-2 (SARS-CoV-2) that causes coronavirus disease 2019 (COVID-19), first appeared in Wuhan (China) and the COVID-19 pandemic spread rapidly around the world. ${ }^{6-8}$ The first case in Turkey was recorded on 11 March and following this many hospitals have been turned into the COVID-19 pandemic hospitals and elective surgical procedures and non-critical health care services are limited. The lockdown has also made it difficult for patients to access healthcare services for nonCOVID-19 conditions in addition to healthcare limitations. Many studies revealed that hospital admission for acute medical illnesses, including stroke and acute myocardial infarction, fell dramatically with the onset of the COVID-19 pandemic. ${ }^{9-11}$

In this study, we aimed to determine the duration of hospital admission of the patients with panic diagnosis pre-COVID-19 and during the COVID-19 pandemic, to examine the factors that may influence hospital admission time, and to identify the impact of the COVID-19 pandemic on hospital admission time.

\section{Material and Methods}

This study was conducted according to the Declaration of Helsinki's principles. The medical ethics committee (Approval No. 22.09.2020/09/09/01) approved this study. We evaluated the Erzincan Mengücek Gazi Training and Research Hospital (EMGTRH), Pathology Department records and determined the patients with a panic diagnosis between January 2018 and January 2021. Patients who had inappropriate contact information in the hospital information processing system were excluded from the study. We reviewed patients' records and documented demographic, clinical, and panic diagnoses from data. We divided the cases into two groups according to the date of their panic diagnosis. Cases diagnosed before 11 Mach 2020 were included in the pre-COVID group and the cases diagnosed afterward 11 Mach 2020 were included in the COVID pandemic group.

We determined the date of admission to the hospital of the patients after receiving panic diagnosis notification through the hospital system and then compared notification and admission date to determine the patients' admission to the hospital time.

Patients were divided into two groups according to the median of the patient's admission time. The applicants within five business days after receiving notification were assigned to a fast group (FG), whereas later application was considered as in slow group (SG). We evaluated the variables (age, gender, the distance of the patient home to hospital, and verbal notification status) that we considered likely to affect the hospital admission time in these groups.

Statistical analysis was performed using SPSS version 15. Descriptive statistics were presented as mean and standard deviation, median, and distribution width. Comparison of continuous variables between groups was conducted using Student's t-test and Mann-Whitney U test according to their distribution. Also, a chi-square test was used for risk estimation. The confidence level for statistical significance was defined as 95 percent $(\alpha=0.05)$.

Panic (unexpected) diagnosis lists of our department that determined according to the national pathology societies recommend, were presented in Table 1.

\section{Results}

There were 74 cases reported as a panic diagnosis in EMGTRH between January 2018-2021. 9 patients who had inappropriate contact information were excluded from this study. A total of 65 patients were included in this study (Figure 1). The distribution of panic diagnosis of the 65 cases, demographic data, verbally notification status, and reaction time were presented in supplement data 1. 
Of these 65 cases, 23 were males, and 42 were females; the median age was 52 years [range, 10-85]. One patient had leukocytoclastic vasculitis, 10 patients had uterine contents without villi or trophoblasts, and 54 patients had unexpected malignancy. The distribution of cases that had unexpected malignancy according to diagnosis was presented in Figures 2-3.

There were 55 cases in the pre-COVID group. Thirty cases' hospital admission times were five days or fewer when cases were recruited into FG, and 25 cases' hospital admission time was longer than five days when cases were recruited into SG. The average length of admission to the hospital was 2.2 days in FG and was 99 days in SG (7-360). The average age was 47 years in FG and 59 years in SG. The average distance of the patient's living area to the hospital was $11 \mathrm{~km}$ (1-52) in the FG and $59 \mathrm{~km}$ in SG (4-390 km).

There was a statistically significant difference in the average distance of the patient's living area to the hospital, age, and notification status between FG and SG in the pre-COVID group. There was no statistically significant difference in gender between FG and SG. The summary of distribution and statistical comparison of age and distance between patient home and hospital among FG and SG are presented in Table 2.

Among pre-pandemic group cases, forty were verbally informed about panic diagnosis by phone call, 15 were not able to inform due to wrong phone number records. The mean time (day) of admission to the hospital of verbally informed and not verbally informed cases were five days and 156 days, respectively. Our results revealed that receiving verbal phone notification was significantly associated with patients' admission to the hospital time (Table 3). Admission times were on mean about 151 days longer in a patient in the not verbally informed cases compared to verbally informed cases in pre-COVID group.

There were 10 cases in the COVID pandemic group. Four cases admission times were five days or fewer (1-5) and six cases admission time was longer than five days (16-40). We ascertained that four cases in the COVID pandemic group were receiving treatment in the home due to COVID-19 infection at the time of diagnosis.

All cases in the COVID pandemic group were verbally informed about panic diagnosis by phone call. The mean time (day) of admission to the hospital was 18.3 days (1-40). Admission times were on mean about 13.3 days longer in verbally informed cases in the COVID pandemic group compared to verbally informed cases in the pre-COVID group.

\section{Discussion}

The concept of critical value in clinical pathology was first described by Lundberg in 1972 as "Pathophysiological derangements at such variance with normal as to be life-threatening if therapy is not instituted immediately." ${ }^{12}$ The critical values in surgical pathology handled by Pereira et al. approximately thirty years from this, and they described possible surgical pathology critical value cases that need immediate communication. ${ }^{1}$ Over the years, the concept of critical diagnosis has been adopted by pathologists, and communication checklists have been added to Laboratory Accreditation Programs by National Pathology Societies. ${ }^{2}$ National pathology societies recommend that each pathology department should identify potential panic (unexpected) diagnosis lists and draw up a communication policy. ${ }^{2}$

Our panic diagnosis policy has been created according to the national pathology societies recommend; when a panic diagnosis is detected, verbal communication provides with the patient's responsible clinicians as soon as possible. The information of the clinicians and the notification time are noted on the panic diagnosis form. When we sign-out a panic diagnosis report, we indicate the patient as a panic diagnosed patient over the hospital information processing system (HIPS). Subsequently, the HIPS sends a notification message to the system and cell phone of the responsible clinician. The HIPS also sends an information message to the patient's phone. We attach importance to informing the responsible clinicians as well as informing the patients verbally in the panic diagnosis notification. The patient is only informed about the pathology report is ready and to admission to the hospital as soon as possible. No information is given about the diagnosis.

Most of the panic diagnosis cases were detected in materials sent from the surgical services department and these clinicians devote most of their employment period to surgical procedures. If clinicians receive the panic 
diagnosis notification during surgical procedures, reaching a patient's contact information may take a long time. For this reason, we prefer to provide verbal information to the patient.

The annual average number of cases in our department was approximately 12000 and panic diagnosis cases accounted for approximately $0.25 \%$ of them. We recorded a significant decrease in the number of cases during the COVID pandemic. Studies showed that panic diagnosis rates accounted for $0.5-20 \%$ of all cases. ${ }^{13,14}$ This rate may differ according to the specific institutional factors, such as the bed capacity, the organ transplantation unit, and case types. Informing patients verbally about the diagnosis can cause a serious increase in the daily workload in centers with a high panic diagnosis reporting rate.

Several studies indicated that well-timed effective verbal communication had a beneficial impact on patient's outcome and treatment management. ${ }^{5,13}$ Staats et al. revealed that pathology laboratories had different approaches to time limitation, such as within 1-hour, same day, or no specific time frame, for communicating with the clinician. ${ }^{15}$ We do not have a strict time frame policy. Most of our cases had unexpected malignancy diagnoses and the information content is more important than the time of communication. Therefore, we provide communication between clinicians and pathologists as soon as possible. Our findings showed that the duration of admission to the hospital of panic diagnosed patients varied between 1 and 360 days. The prolonged admission time indicates that patients are not adequately informed about following up pathology reports, even if only indirectly.

The most important findings of our study were taking a phone notification has a beneficial impact on admission time. Admission times were on mean about 151 days longer in the patient in the not verbally informed group compared to the verbally informed group in the pre-COVID period. We observed that even if the patients were verbally informed during the COVID pandemic, they applied to the hospital for a longer period compared to the pre-pandemic period, five days and 18.3 days, respectively.

We could not make a notification to fifteen patients since the contact information in HIPS belonged to different people or was not up to date. We believe that Informing patients about the process of pathology reports and reminding them to keep their phone numbers in hospital records up to date to communicate in possible panic diagnosis situations may help shorten the admission time.

Many studies revealed that hospital admission for acute medical illnesses, including stroke and acute myocardial infarction, fell dramatically with the onset of the COVID-19 pandemic. ${ }^{9-11}$ The most reasonable explanation for patients' attitude is that the limitation of elective surgical procedures and non-critical health care services and quarantine procedure made it difficult for patients to access healthcare services for nonCOVID-19 conditions or patients avoided seeking hospital care, perhaps in response to the fear of COVID19 infection. Transportation of patients with COVID-19 to the hospital is provided only through the 112 Emergency Ambulance Service (EAS) in Turkey. EAS evaluates the patient's complaints related to infection and decides for the transportation of patients with COVID-19 to the hospital. Informing the EAS about the provision of transportation to the hospital in cases of COVID-19 positive panic diagnosed patients may be effective in shortening the admission time.

In Turkey, doctors and patients can access health data collected from the health institution, regardless of where the examinations and treatments are held, via e-nabiz that is an application developed by the Ministry of Health. Cell phone applications such as e-nabiz that provide communication between patients and the health care system, contribute positively to the country's health care system. In our country, primary care can also reach patients in a brief time via e-nabiz. Therefore, we believe that sending automatic messages to family medicine units, which are primarily responsible for patients with applications such as e-nabız, can increase the chance of success in reaching the patient in cases of panic diagnosis. Our hospital has been integrated into this system in 2020. Due to the small sample size, the effect of this system on the application period cannot be evaluated clearly.

So far, a limited number of studies have been published on panic diagnosis. Most of the previous studies focused on the general recommendation of critical value policy, effective communication of critical diagnosis, or documentation of possible diagnostic list considered a critical diagnosis by pathologist or clinician. ${ }^{13,14,16-20}$ 
To the best of our knowledge, this study is the first attempt at a comprehensive evaluation of factors that may affect the time of admission to the hospital who reach a panic diagnosis. Our study has some limitations. This study has retrospective character in one single center and only provides information about the duration of hospital admission and trends of patients living around Erzincan. Therefore, our findings cannot be generalized to the other population. Nevertheless, we believe that the findings of this study may be helpful to review the panic diagnosis communication policies of pathology laboratories. Further research with wellplanned multi-centric studies in larger patient groups may be helpful to contribute to the development of panic diagnosis policy.

\section{Conclusion}

Several studies indicated that well-timed effective verbal communication had a beneficial impact on patient outcomes and treatment management. Our findings revealed that patients who were verbally informed about panic diagnosis were admitted to the hospital in a shorter time. Therefore, we believe that informing patients verbally should be included in panic diagnosis policies, patients should be informed about the follow-up of the pathology report and their contact information should be kept up to date. Besides, integrations of hospital panic diagnosis notification systems to health application programs and primary responsible family physician's systems may be useful for preventing unwanted delays.

\section{Notes}

Conflict of interest: None of the authors received any type of financial support that could be considered potential conflict of interest regarding the manuscript or its submission.

Funding : The author(s) received no financial support for the research, authorship, and/or publication of this article.

\section{References}

1. Pereira TC, Liu Y, Silverman JF. Critical values in surgical pathology. Am J Clin Pathol. 2004; 122: 201-5. doi: 10.1309/7NRW-7G68-4VEP-WPMR.

2. Nakhleh RE, Myers JL, Allen TC, et al. Consensus statement on effective communication of urgent diagnoses and significant, unexpected diagnoses in surgical pathology and cytopathology from the College of American Pathologists and Association of Directors of Anatomic and Surgical Pathology. Arch Pathol Lab Med. 2012; 136: 148-54. doi: 10.5858/arpa.2011-0400-SA.

3. LiVolsi VA. Critical values in anatomic pathology: how do we communicate? Am J Clin Pathol. 2004; 122: 171-2. doi: 10.1309/G1C5-1DYV-MPDJ-3DD7.

4. Jackson C, Macdonald M, Anderson M, Stevens P, Gordon P, Laxer R. Improving communication of critical test results in a pediatric academic setting: key lessons in achieving and sustaining positive outcomes. Healthc Q. 2009; 12: 116-22. doi: 10.12927/hcq.2009.20978.

5. Georgiou A, Li J, Thomas J, Dahm MR, Westbrook JI. The impact of health information technology on the management and follow-up of test results - a systematic review. J Am Med Inform Assoc. 2019; 26: 678-88. doi: 10.1093/jamia/ocz032.

6. Hui DS, I Azhar E, Madani TA, et al. The continuing 2019-nCoV epidemic threat of novel coronaviruses to global health - The latest 2019 novel coronavirus outbreak in Wuhan, China. Int J Infect Dis. 2020; 91: 264-6. doi: 10.1016/j.ijid.2020.01.009.

7. Zhu N, Zhang D, Wang W, et al. China Novel Coronavirus Investigating and Research Team. A Novel Coronavirus from Patients with Pneumonia in China, 2019. N Engl J Med. 2020; 382: 727-33. doi: 10.1056/NEJMoa2001017.

8. Coronaviridae Study Group of the International Committee on Taxonomy of Viruses. The species Severe acute respiratory syndrome-related coronavirus: classifying $2019-\mathrm{nCoV}$ and naming it SARSCoV-2. Nat Microbiol. 2020; 5: 536-44. doi: 10.1038/s41564-020-0695-z.

9. Birkmeyer JD, Barnato A, Birkmeyer N, Bessler R, Skinner J. The Impact Of The COVID-19 Pandemic On Hospital Admissions In The United States. Health Aff (Millwood). 2020; 39: 2010-7. doi: 10.1377/hlthaff.2020.00980. 
10. Siegler JE, Heslin ME, Thau L, Smith A, Jovin TG. Falling stroke rates during COVID-19 pandemic at a comprehensive stroke center. J Stroke Cerebrovasc Dis. 2020; 29: 104953. doi: 10.1016/j.jstrokecerebrovasdis.2020.104953.

11. Solomon MD, McNulty EJ, Rana JS, et al. The Covid-19 Pandemic and the Incidence of Acute Myocardial Infarction. N Engl J Med. 2020; 383 (7): 691-3. doi: 10.1056/NEJMc2015630.

12. Lundberg GD. Panic values: five years later. MLO Med Lab Obs. 1977; 9: 27-34

13. Association of Directors of Anatomic and Surgical Pathology. Critical diagnoses (critical values) in anatomic pathology. Am J Surg Pathol. 2006; 30: 897-9. doi: 10.1097/01.pas.0000213287.73530.0a.

14. Huang EC, Kuo FC, Fletcher CD, Nosé V. Critical diagnoses in surgical pathology: a retrospective single-institution study to monitor guidelines for communication of urgent results. Am J Surg Pathol. 2009; 33: 1098-102. doi: 10.1097/PAS.0b013e3181a05ea9.

15. Staats PN, Parkash V, Otis CN, Sharma P, Ioffe O, Bracamonte ER. The Current State of Communication of Urgent and Significant, Unexpected Diagnoses in Anatomic Pathology. Arch Pathol Lab Med. 2020; 144: 1067-74. doi: 10.5858/arpa.2019-0436-OA.

16. Pereira TC, Clayton AC, Tazelaar HD, Liu Y, Leon M, Silverman JF. Critical values in cytology. Diagn Cytopathol. 2006; 34: 447-51. doi: 10.1002/dc.20443.

17. Pereira TC, Silverman JF, LiVolsi V, et al. A multi-institutional survey of critical diagnoses (critical values) in surgical pathology and cytology. Am J Clin Pathol. 2008; 130: 731-5. doi: 10.1309/AJCPEHGNFGBEJZ7H.

18. Nakhleh RE, Souers R, Brown RW. Significant and unexpected, and critical diagnoses in surgical pathology: a College of American Pathologists' survey of 1130 laboratories. Arch Pathol Lab Med. 2009; 133: 1375-8. doi: 10.1043/1543-2165-133.9.1375.

19. Dighe AS, Rao A, Coakley AB, Lewandrowski KB. Analysis of laboratory critical value reporting at a large academic medical center. Am J Clin Pathol. 2006; 125: 758-64. doi: 10.1309/R53X-VC2U-5CH6TNG8.

20. Etchells E, Adhikari NK, Cheung C, et al. Real-time clinical alerting: effect of an automated paging system on response time to critical laboratory values-a randomised controlled trial. Qual Saf Health Care. 2010; 19: 99-102. doi: 10.1136/qshc.2008.028407.

Table 1 Panic diagnosis List of EMG TARH pathology department

Cases with immediate clinical consequences Leukocytoclastic vasculitis

Uterine contents without villi or trophoblast

Fat in an endometrial curettage specimen

Fat in colonic endoscopic polypectomy specimens

Unexpected or discrepant findings

Unexpected or discrepant findings

Significant disagreement between frozen section and final

diagnoses

Significant disagreement between immediate interpretation

and final FNA diagnosis

Unexpected malignancy

Significant disagreement and/or change between diagnoses of primary pathologist and outside pathologist consultation (at the original or consulting institution) 


\begin{tabular}{ll}
\hline Cases with immediate clinical consequences & Leukocytoclastic vasculitis \\
& Uterine contents without villi or trophoblast \\
& Fat in an endometrial curettage specimen \\
& Fat in colonic endoscopic polypectomy specimens
\end{tabular}

\section{Infections}

Bacteria or fungi in cerebrospinal fluid cytology in immunocompromise Pneumocystis organisms, fungi, or viral cytopathic changes in bronchoalveolar lavage, bronchial washing, or brushing cytology specimens in immunocompromised or immunocompetent pati Acid-fast bacilli in immunocompromised or immunocompetent patients Fungi in FNA specimen of immunocompromised patients Bacteria in heart valve or bone marrow

Herpes in Papanicolaou smears of near-term pregnant patients Any invasive organism in surgical pathology specimens of immunocompromised patients

\begin{tabular}{lll}
\hline & Fast Admitted Group Fast Admitted Group Slow Ad & $\begin{array}{l}\text { Fad } \\
\text { Median (Min-Max) }\end{array}$ \\
Mean $\pm \mathbf{S}$ & Mean \pm SD & $48,50(10,00-79,00)$ \\
Age & $46,83 \pm 17,86$ & $59 \pm 16,05$ \\
The distance between patient home and hospital & $10,03 \pm 13,02$ & $5,00(1,00-52,00)$ \\
\hline
\end{tabular}

SD: Standard deviation

1 Student's t test, statistically significant at 0.95 confidence level

${ }^{2}$ Mann-Whitney U test, Statistically significant at 0.999 confidence level

Table 2 The distribution and statistical comparison of age and distance between patient home and hospital among groups in pre- COVID period.

\begin{tabular}{|c|c|c|c|c|c|}
\hline & & FG & SG & $\mathbf{p}$ & OR $(95 \% \mathrm{CI})$ \\
\hline Gender & Male/Female & $7 / 23$ & $12 / 13$ & $0,138^{*}$ & $0,431(0,140-1,326)$ \\
\hline Notification Status & Not Verbally informed /Verbally informed & $0 / 30$ & $15 / 10$ & $<0,001^{* *}$ & $\mathrm{~N} / \mathrm{A}$ \\
\hline
\end{tabular}

*not statistically significant $(\mathrm{p}>0.05)$

**Statistically significant at 0.999 confidence level

OR: Odds ratio, FG: Fast admitted Group, SG: Slow Admitted Group

Table 3 Chi-square test results between study groups in pre-COVID period, notification status, and gender.

Panic Diagnosed Patient pre-COVID period

\begin{tabular}{lllll}
\hline No & Gender & Age & Preliminary diagnosis & Procedure \\
1 & M & 64 & Vertebra fracture & Vertebral bone curettage \\
2 & W & 38 & Multinodular goiter & Thyroidectomy \\
3 & M & 78 & Benign prostate hyperplasia & Transurethral resection \\
4 & W & 60 & Leukoclastic vasculitis & Punch biopsy \\
5 & M & 61 & Pangastritis & Endoscopic biopsy \\
6 & W & 44 & Acute appendicitis & Appendectomy \\
7 & W & 40 & Menorrhagia, Polyp & Curettage
\end{tabular}

Pathological diagnosis
Multiple myeloma
Papillary microcarcinoma
Prostatic adenocarcinoma
Leukoclastic vasculitis
Adenocarcinoma
Neuroendocrine neoplasm
Squamous cell carcinoma




\begin{tabular}{|c|c|c|c|c|c|}
\hline 8 & $\mathrm{~W}$ & 52 & Screen test & SMEAR & HSIL \\
\hline 9 & $\mathrm{~W}$ & 55 & Menorrhagia, Polyp & Curettage & Endometrial carcinoma \\
\hline 10 & $\mathrm{~W}$ & 59 & Myoma uteri & $\mathrm{TAH}+\mathrm{BSO}$ & Serous carcinoma of tuba uterine \\
\hline 11 & W & 79 & Menorrhagia, Polyp & Curettage & Squamous cell carcinoma \\
\hline 12 & $\mathrm{~W}$ & 10 & Pyogenic granuloma & Lesion excision & Atypical spitz nevus \\
\hline 13 & $\mathrm{~W}$ & 22 & Suspicious of an ectopic pregnancy & Curettage & Uterine contents without villi \\
\hline 14 & $\mathrm{~W}$ & 25 & Suspicious of an ectopic pregnancy & Curettage & Uterine contents without villi \\
\hline 15 & M & 64 & Benign prostate hyperplasia & Transurethral resection & Prostatic adenocarcinoma \\
\hline 16 & $\mathrm{~W}$ & 32 & Suspicious of an ectopic pregnancy & Curettage & Uterine contents without villi \\
\hline 17 & $\mathrm{~W}$ & 28 & Suspicious of an ectopic pregnancy & Curettage & Uterine contents without villi \\
\hline 18 & $\mathrm{~W}$ & 26 & Suspicious of an ectopic pregnancy & Curettage & Uterine contents without villi \\
\hline 19 & W & 30 & Suspicious of an ectopic pregnancy & Curettage & Uterine contents without villi \\
\hline 20 & W & 27 & Suspicious of an ectopic pregnancy & Curettage & Uterine contents without villi \\
\hline 21 & W & 29 & Suspicious of an ectopic pregnancy & Curettage & Uterine contents without villi \\
\hline 22 & $\mathrm{~W}$ & 50 & Multinodular goiter & Thyroidectomy & Papillary microcarcinoma \\
\hline 23 & W & 42 & Multinodular goiter & Thyroidectomy & Papillary microcarcinoma \\
\hline 24 & M & 73 & Multinodular goiter & Thyroidectomy & Papillary microcarcinoma \\
\hline 25 & $\mathrm{~W}$ & 66 & Multinodular goiter & Thyroidectomy & Papillary microcarcinoma \\
\hline 26 & M & 49 & Multinodular goiter & Thyroidectomy & Papillary microcarcinoma \\
\hline 27 & $\mathrm{~W}$ & 54 & Myoma uteri & $\mathrm{TAH}+\mathrm{BSO}$ & Endometrial carcinoma \\
\hline 28 & $\mathrm{~W}$ & 48 & Myoma uteri & $\mathrm{TAH}+\mathrm{BSO}$ & Endometrial carcinoma \\
\hline 29 & M & 62 & Acute appendicitis & Appendectomy & Neuroendocrine neoplasm \\
\hline 30 & $\mathrm{~W}$ & 38 & Acute appendicitis & Appendectomy & Low-grade mucinous neoplasm \\
\hline 31 & M & 67 & Lipoma & Lesion excision & Metastatic Squamous cell carcinoma \\
\hline 32 & $\mathrm{~W}$ & 81 & Vaginitis & SMEAR & HSIL \\
\hline 33 & $\mathrm{~W}$ & 85 & Pangastritis & Endoscopic biopsy & Adenocarcinoma \\
\hline 34 & M & 61 & Pangastritis & Endoscopic biopsy & Adenocarcinoma \\
\hline 35 & $\mathrm{~W}$ & 64 & Myoma uteri & $\mathrm{TAH}+\mathrm{BSO}$ & Squamous cell carcinoma \\
\hline 36 & $\mathrm{~W}$ & 41 & Myoma uteri & $\mathrm{TAH}+\mathrm{BSO}$ & Endometrial carcinoma \\
\hline 37 & $\mathrm{~W}$ & 65 & Multinodular goiter & Thyroidectomy & Papillary microcarcinoma \\
\hline 38 & W & 73 & Pyogenic granuloma & Lesion excision & Malign Melanoma \\
\hline 39 & M & 65 & Benign prostate hyperplasia & Transurethral resection & Prostatic adenocarcinoma \\
\hline 40 & M & 67 & Benign prostate hyperplasia & Transurethral resection & Prostatic adenocarcinoma \\
\hline 41 & M & 70 & Benign prostate hyperplasia & Transurethral resection & Prostatic adenocarcinoma \\
\hline 42 & M & 66 & Benign prostate hyperplasia & Transurethral resection & Prostatic adenocarcinoma \\
\hline 43 & $\mathrm{~W}$ & 58 & Multinodular goiter & Thyroidectomy & Papillary microcarcinoma \\
\hline 44 & $\mathrm{~W}$ & 43 & Multinodular goiter & Thyroidectomy & Papillary microcarcinoma \\
\hline 45 & M & 60 & Pangastritis & Endoscopic biopsy & Neuroendocrine neoplasm \\
\hline 46 & $\mathrm{~W}$ & 69 & Multinodular goiter & Thyroidectomy & Papillary microcarcinoma \\
\hline 47 & M & 50 & Pilonidal cyst & Lesion excision & Malign Melanoma \\
\hline 48 & $\mathrm{~W}$ & 56 & Screen test & SMEAR & HSIL \\
\hline 49 & M & 36 & Acute appendicitis & Appendectomy & Low-grade mucinous neoplasm \\
\hline 50 & M & 70 & Acute appendicitis & Appendectomy & Metastatic Adenocarcinoma \\
\hline 51 & $\mathrm{~W}$ & 80 & Acute appendicitis & Appendectomy & Low-grade mucinous neoplasm \\
\hline 52 & M & 29 & Acute appendicitis & Appendectomy & Low-grade mucinous neoplasm \\
\hline 53 & $\mathrm{~W}$ & 40 & Acute appendicitis & Appendectomy & Low-grade mucinous neoplasm \\
\hline 54 & $\mathrm{~W}$ & 23 & Acute appendicitis & Appendectomy & Neuroendocrine neoplasm \\
\hline 55 & M & 56 & Acute appendicitis & Appendectomy & Mucinous Adenocarcinoma \\
\hline
\end{tabular}

Panic diagnosed patient during COVID pandemic 


\begin{tabular}{llll} 
No & Gender & Age & Preliminary diagnosis \\
1 & W & 55 & Multi nodular goiter \\
2 & W & 53 & Vaginitis \\
3 & W & 29 & Suspicious of an ectopic pregnancy \\
4 & W & 27 & Suspicious of an ectopic pregnancy \\
5 & $\mathrm{M}$ & 71 & Benign prostate hyperplasia \\
6 & $\mathrm{w}$ & 53 & Menorrhagia, Polyp \\
7 & $\mathrm{M}$ & 45 & Multi nodular goiter \\
8 & $\mathrm{~W}$ & 40 & Acute appendicitis \\
9 & $\mathrm{M}$ & 55 & Viral pneumonia \\
10 & $\mathrm{M}$ & 70 & Orchitis \\
\hline
\end{tabular}

Procedure

Thyroidectomy

SMEAR

Curettage

Curettage

Transurethral resection

Curettage

Thyroidectomy

Appendectomy

Thoracentesis

Orchiectomy
Pathological diagnosis

Papillary microcarcinoma

Atypical glandular cells

Uterine contents without villi

Uterine contents without villi

Prostatic adenocarcinoma

Squamous cell carcinoma

Papillary microcarcinoma

Low-grade mucinous neoplasm

Lung Adenocarcinoma

Lymphoma
Adm

$30 \mathrm{~d}$

$16 \mathrm{~d}$

3 day

2 day

$36 \mathrm{~d}$

1 day

$25 \mathrm{dc}$

$40 \mathrm{dc}$

5 das

$25 \mathrm{~d}$

W: Woman; M; Man; TAH+BSO: Total abdominal hysterectomy with bilateral salpingo-oophorectomy; FG: Fast Group; SG: Slow Group; P: verbally informed; N: not verbally informed.

Supplement data-1 Demographic data, verbally notification status, admission to the hospital time and clinicopathological characteristic of the patients.

Figure 1 Flowchart demonstrating excluded cases and distribution of included cases according to hospital admission time.

Figure 2 Distribution of cases according to causes of panic diagnosis (a) Distribution of cases with unexpected malignancy (b).

Figure 3 a; Leukocytoclastic vasculitis (H\&E x100), b; Uterine contents without villi or trophoblasts (H\&E x40), c; Papillary microcarcinoma (H\&E x40), d; Low-grade mucinous neoplasm (H\&E x200), e; Endometrial polyp and endometrial carcinoma (H\&E x100), f; Malign melanoma (H\&E x200).

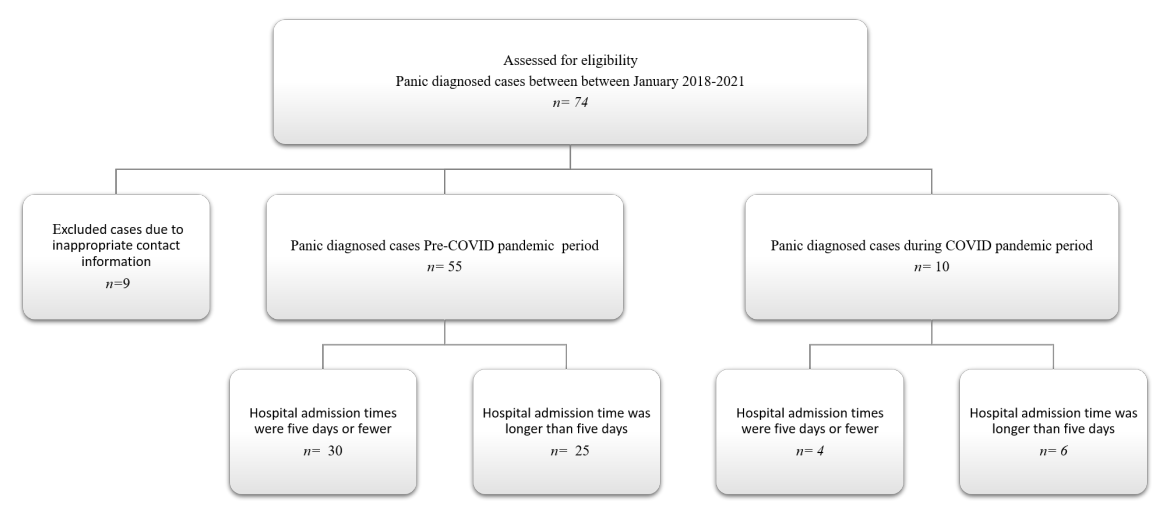




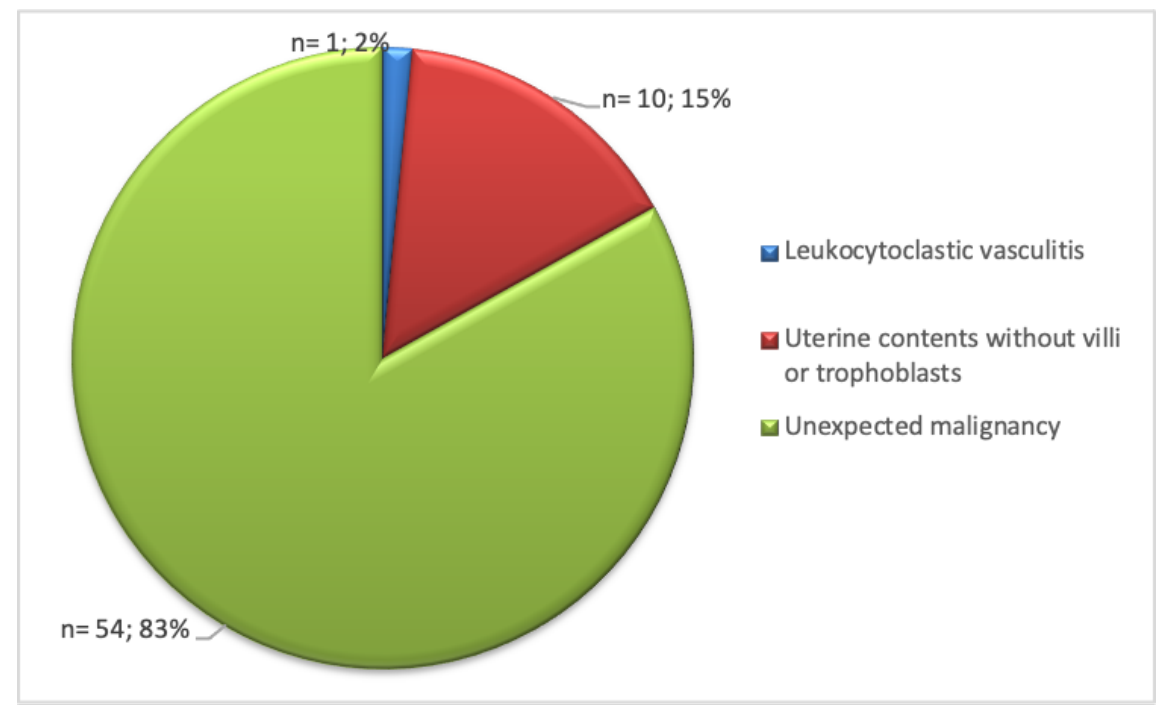

Distribution of the unexpected malignancy cases

Papillary microcarcinoma in thyroidectomy

LMAN, neuroendocrine tumor and metastatic adenocarcinoma.. Prostatic adenocarcinoma in Transurethral resection

Endometrial, Tubal and Squamous cell carcinoma in hysterectomy HSIL and AGUS in SMEAR

Malignant melanoma, Atypical spitz nevus and metastatic. Adenocarcinoma and neuroendocrine tumor in endoscopic biopsy Squamous cell carcinoma and endometrial adenocarcinoma in

Adenocarcinoma in pleural fluid

Mulltiple myeloma in vertebral bone fracture

Lymphoma in orchiectomy
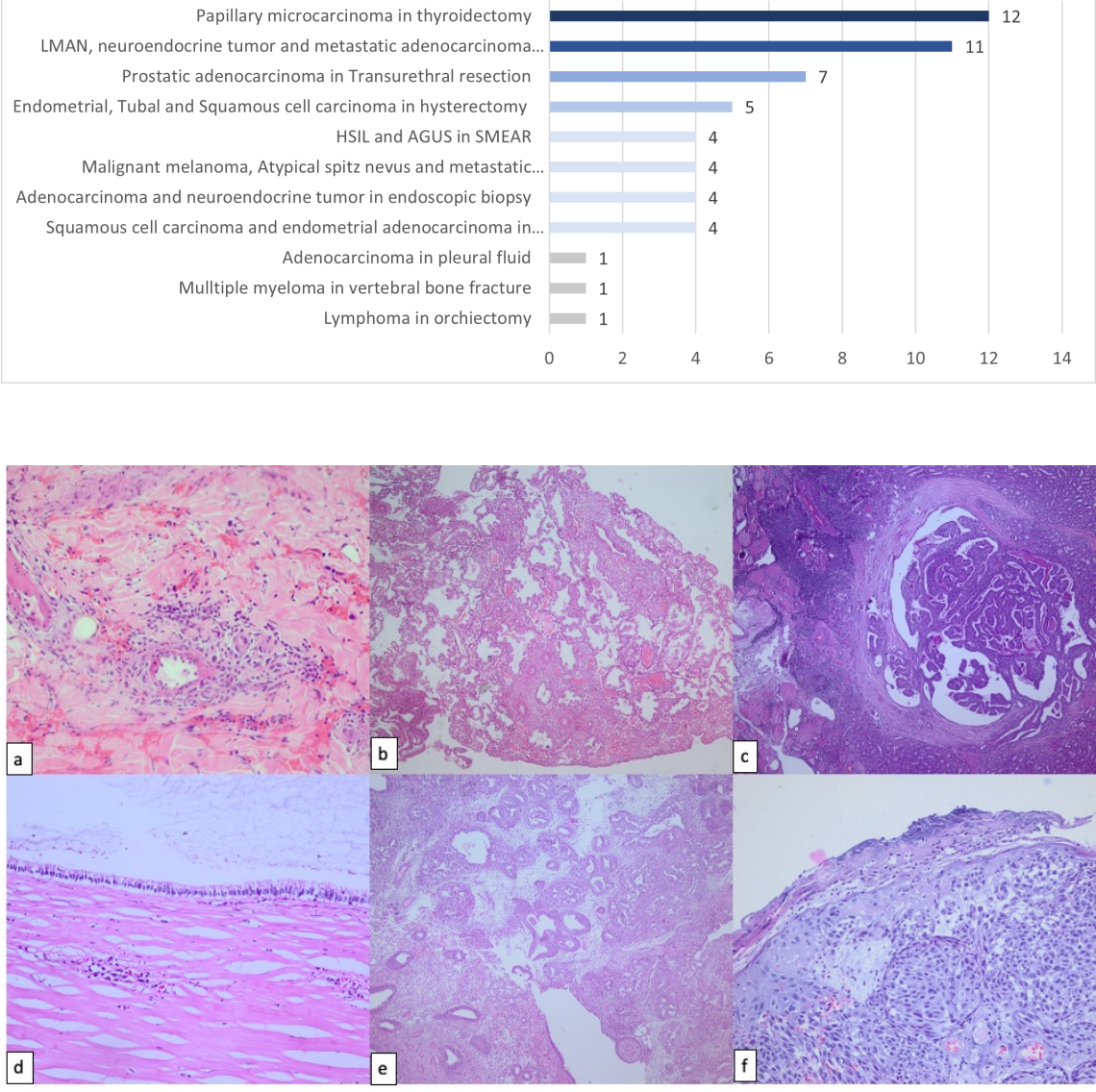